\title{
ZUR VORGESCHICHTE DER DÄNISCHEN IAA-SEKTION
}

1971 konnte das 100-jährige Bestehen der dänischen Arbeiterbewegung gefeiert werden. Ihre Geschichte begann als die Geschichte der dänischen Sektion in der Internationalen Arbeiterassoziation: noch bevor nämlich der Internationale Arbeiterverein in Dänemark (Den internationale Arbejderforening $i$ Danmark) von seinem Gründer, dem Reserveleutnant, Hauslehrer und ehemaligen Postschreiber Louis Albert François Pio ein festes Organisationsgebäude verschrieben bekam, hatte dieser Kontakt mit Marx in London aufgenommen und ihm in einem Brief die politischen Ziele und programmatischen Vorstellungen der noch zu konstituierenden dänischen Sektion der IAA annonciert (18. September 1871). ${ }^{1}$ Die Selbstverständlichkeit, mit der der erst am 15. Oktober $1871^{2}$ mit einem organisatorischen Dach versehene dänische Arbeiterverein der IAA als dänische Sektion angekündigt wurde, lässt vorbereitende Kontakte zwischen London und Kopenhagen vermuten. Es darf angenommen werden, dass Pio und die Gefolgsleute der ersten Stunde feste Vorstellungen über die theoretischen und organisatorischen Grundlagen der IAA und einzelner Sektionen als Richtschnur für ihr Unternehmen gehegt haben müssen, kurz: dass ihnen die internationale Arbeiterbewegung ein Begriff war. Dafür sprechen auch Pios erste schriftstellerischen Versuche im Dienste des Sozialismus: die Socialistiske Blade I und II $^{3}$ mögen noch so dilettantisch gewesen sein - die Absicht, die Lage der dänischen Unterklasse mit Hilfe der sozialistischen Theorie zuerst zu definieren, dann die Betroffenen zu organisieren, wird erkenntlich. Ohne Informationen, ohne Kontakte zur IAA hätte Pio jedoch kaum einen Buchstaben zu Papier bringen können.

1 Pio an Marx, 18.9.1871, in: Børge Schmidt (ed.), 80 Louis Pio Breve og en Pio-Bibliografi, Kopenhagen 1950, S. $25 \mathrm{ff}$.

2 O. Bertolt, E. Christiansen, P. Hansen, En Bygning vi rejser. Den politiske Arbejderbevægelses historie i Danmark, Bd 1, Kopenhagen 1954, S. 58.

3 En Arbejder [Louis Pio], Socialistiske Blade i tvangfri Hefter, I: Socialismen i dens Forhold til Historien, II : Hvad vi forlanger, beide Kopenhagen 1871. 
Welche Kontakte waren Pios Socialistiske Blade nun vorausgegangen, woher nahm der Verfasser sein Wissen? Die Literatur zur dänischen Arbeiterbewegung vermag diese Frage nicht zu beantworten. Mit der Ausnahme des Besuches von Lorents Petersen im Frühjahr 1870 in Kopenhagen, von dem noch die Rede sein wird, ${ }^{1}$ ist über die Vorgeschichte der dänischen Arbeiterbewegung nichts bekannt. Die Geschichtsschreibung behilft sich stattdessen mit Vermutungen ${ }^{2}$ und lässt im übrigen den von Oluf Bertolt, ${ }^{3}$ dem ersten (dänischen) Forscher, der sich ernsthaft mit der Vorgeschichte der dänischen IAASektion beschäftigte, aufgestellten Satz, dass ,,irgendeine direkte Verbindung zwischen Arbeitern in Dänemark und Marxens Internationale vor dem Frühjahr 1870 nicht festgestellt werden kann", stillschweigend gelten.

Vor diesem Hintergrund bleibt die Tat des Louis Pio, die Gründung der dänischen Arbeiterbewegung als IAA-Sektion, sowie die nicht unbeträchtliche Resonanz unter denen, deren es habhaft zu werden galt - Pio berichtete Marx von ca. 700 Arbeitern, die er schon für seine Sache"gewonnen habe ${ }^{5}$ - letzlich unerklärbar. Sie ist so nur deutbar als Überraschungserfolg eines selbstgenügsamen Genies, welches Vorbilder ebensowenig benötigte wie Inspirationen. Genauso zeichnet die vorwiegend dänische Literatur denn auch ihr Bild von der Gründung der dänischen IAA-Sektion sowie von deren Gründer. ${ }^{6}$ Dass es so nicht war, sondern dass im Gegenteil zwar nicht zahlreiche, so doch kontinuierliche Kontakte zwischen Dänemark und der IAA bestanden haben, und dass es Männer gab, die sich ernsthaft um diese Kontakte bemühten, soll auf den folgenden Seiten nachgewiesen werden. Neben schon seit längerem gedruckt vorliegendem, aber noch nicht ausgewertetem Material (Minutes) waren es vor allem einige kleinere Archivfunde, die diese Arbeit möglich machten und dabei halfen, bisher Vermutetes zu untermauern und Indizienketten $\mathrm{zu}$ erstellen, die geeignet sein sollten, neues Licht auf eine bisher weitgehend unaus-

1 Gustav Jaeckh, Die Internationale. Eine Denkschrift zur vierzigjährigen Gründung der Internationalen Arbeiter-Assoziation, Leipzig 1904, S. 229 f.

2 So zuletzt Willy Markvad, "Arbejderforeninger i Danmark 1848-1871", in : Årbog for Arbejderbevægelsens historie, 1. Jahrg. (1971), S. 7-45; 31.

3 Oluf Bertolt (1891-1958) war Autodidakt. Der gelernte Maler leitete zeitweilig das ABA (Arbejderbevægelsens Bibliotek og Arkiv) in Kopenhagen.

4 Oluf Bertolt, Pionerer. Mændene fra Halvfjerdsernes Arbejderbevægelse, Kopenhagen 1938, S. 27: „Nogen direkte Forbindelse mellem Arbejdere i Danmark og Marx' Internationale kan ikke fastslaas før i foraaret 1870."

5 Siehe S. 54, Anm. 1

- Die Literatur zur dänischen Arbeiterbewegung ist nicht sehr umfangreich; als "Hauptwerke" gelten noch immer die populärwissenschaftlichen Darstellungen „Pionerer” und „En Bygning vi rejser” (siehe oben). 
geforschte Periode in der Geschichte der dänischen Arbeiterbewegung zu werfen.

\section{Ein Däne (selbst Schneider)}

Der erste Däne, dessen Mitgliedschaft in der IAA bezeugt ist, war ein gewisser N. P. Hansen. Über diesen Mann ist wenig bekannt; es war weder auszumachen, welche Vornamen sich hinter den Initialen $\mathrm{N}$. und $P$. verbergen, noch, wann und wo Hansen geboren wurde und starb. Gesichert ist immerhin, dass er am 13. Dezember 1864 dänisches Mitglied des provisorischen Zentralkommitees der IAA wurde ${ }^{1}$ und dass er von Beruf Schneider war. 1866 schickte ihn der Generalrat der IAA auf Agitationsreise nach Edinburgh: gemeinsam mit einem anderen IAA-Agenten, seinem deutschen Berufskollegen Haufe, sollte er den streikenden schottischen Schneidergesellen zu Hilfe kommen, deren Ausstand von dänischen und deutschen Streikbrechern gefährdet wurde. Schon am 27. März 1866 hatte Hansen den Generalrat vom bevorstehenden Export von Streikbrechern nach Schottland ins Bild gesetzt, ${ }^{2}$ und daraufhin scheint der Beschluss gefasst worden zu sein, ihn und Haufe in das Ausstandsgebiet zu schicken. Marx bemerkte dazu in einem Brief an Engels vom 10. Mai 1866, dass der Generalrat „,infolge des deutschen und dänischen Schneiderimports nach Edinburgh einen Deutschen und einen Dänen (beide selbst Schneider) nach Edinburgh geschickt [habe]".3 Auf der Generalratssitzung vom 22. Mai 1866, nach seiner (und Haufes) Rückkehr nach London, berichtete Hansen von der Arbeit in Edinburgh. Seiner Aussage zufolge war sie erfolgreich gewesen. ${ }^{4}$

Einige Zeit später war Hansen dann in Kopenhagen. Das Protokoll der Generalratssitzung vom 18. September 1866 berichtet nämlich:

"Citizen Hansen gave an account of what he had been able to do for the Association during his recent visit to Copenhagen. He stated that he had found there a trustworthy agent for the Association."5

Hansen war also in Kopenhagen gewesen. Die Frage, ob seine Reise in die Stadt, die vielleicht seine Vaterstadt war, nur Besuchen dienen

1 Der Social-Demokrat, 1. Jahrg., Probenummer (30.12.1864); The General Council of the First International, Minutes, Bd I (1864-1866), Moskau und London o.J., S. 55.

2 Ebd., S. 174.

3 Marx, Engels, Werke, Bd 31, Berlin 1965, S. 216.

4 The General Council, a.a.O., S. 194.

5 A.a.O., Bd II (1866-1868), S. $29 f$. 
sollte, oder ob er wieder in offizieller Mission unterwegs war - denkbar wäre der Auftrag des Generalrates, den weiteren Export von dänischen Streikbrechern an Ort und Stelle agitatorisch zu unterlaufen zu versuchen - ist nicht zu beantworten. Die Quellen schweigen darüber. Doch ganz gleich, welchem erklärten Ziel Hansens Reise gedient haben mag: seine Aussage, er habe in Kopenhagen einen trustworthy agent gefunden, bezeugt seinen erfolgreichen Versuch, zwischen Dänemark und der IAA Kontakte herzustellen. Es sind dieses die ersten nachweisbaren Kontakte, die die IAA zu Dänemark hatte.

Das Protokoll der Generalratssitzung vom 18 . September 1866 verrät nichts über die Art dieser Kontakte. Von einem geübten Reiseagitator mit dem Spezialgebiet „dänische Streikbrecher” dürfen wir jedoch annehmen, dass er dieses Thema, wenn es, wie gesagt, nicht gar erklärtes Ziel seiner Reise gewesen war, in jedem Falle angeschnitten hat. Ganz sicher hat Hansen seinen (oder seine) Gesprächspartner auch mit den organisatorischen und theoretischen Prinzipien der IAA bekanntgemacht. Das Protokoll jener Generalratssitzung verschweigt auch den Namen von Hansens Vertrauensmann in Kopenhagen. Wir müssen versuchen, ihn zu erschliessen. Es bietet sich an, diesen Mann unter den Personen zu suchen, die sich im Kopenhagen der 60-er Jahre des 19. Jahrhunderts mit Arbeiterproblemen beschäftigten. Der Kreis der dafür in Betracht kommenden Personen ist jedoch sehr klein, bedingt durch die Tatsache, dass Dänemark noch sehr schwach industrialisiert war und erst die allerersten Anfänge eines Industrieproletariates im Entstehen begriffen waren. ${ }^{1}$ Entsprechend klein war auch der Kreis derjenigen, die sich mit seiner Problematik beschäftigten. Das einzige Milieu im Kopenhagen der 60-er Jahre des 19. Jahrhunderts in dem solche Fragen diskutiert wurden, waren die Kopenhagener Arbeitervereine, ${ }^{2}$ und unter diesen gab es nur einen, der das Muster des liberalen Ideen verpflichteten „Arbeiterbildungsvereines” flexibel genug handhabte, um einem IAA-Gesandten wegen der Verbreitung „französischer Ideen” nicht gleich die Tür zu weisen: den Kopenhagener Arbeiterverein (Kjøbenhavns Arbeiderforening), dessen brillantester Kopf der Landvermesser und Abgeordnete im Folketing Reinholdt Jensen war.

1 Die Frage nach dem Wann und Wie der Industrialisierung Dänemarks hat zu einigem Streit in der dänischen Geschichtswissenschaft und Nationalökonomie geführt. Letzte Forschungsergebnisse und ein Überblick über die ältere Forschung bei Svend Aage Hansen, Early Industrialisation in Denmark, Kopenhagen 1970, vor allem S. 7-11.

2 Siehe dazu Markvads Versuch einer Genealogie der Kopenhagener Arbeitervereine, a.a.O., Beilage II, S. 45 


\section{A trustworthy agent}

Der Kopenhagener Arbeiterverein war zwar der kleinste, aber auch der fortschrittlichste unter mehreren teils berufsständisch-traditionellen, teils liberal eingestimmten Arbeitervereinen der Hauptstadt. Der Verein war 1856 mit 177 Mitgliedern gegründet worden, ${ }^{1}$ hatte sich in den folgenden Jahren immer weiter vom Typus des liberalen Arbeiterbildungsvereines entfernt und auch zu politischen Fragen Stellung bezogen. Darüber kam es 1860 zu einer Sezession, in deren Verlauf der grössere Teil des Vereines als Arbeiterbildungsverein wiedergegründet wurde, während der kleinere, mit kaum mehr als 60 Mitgliedern, unter Beibehaltung des alten Namens den eingeschlagenen Weg konsequent weiterverfolgte ${ }^{2}$ und sich zum radikal-demokratischen Verein aufgeklärter hauptstädtischer Arbeiter und Handwerker fortentwickelte. Massgeblich für diese wichtige Entwicklung war Reinholdt Jensen; er war es, der dem Verein 1865 ein fortschrittliches Programm formulierte, in dem zwar bürgerlich-demokratische Forderungen - vor allem die nach Beibehaltung des beispielhaft liberalen dänischen Junigrundgesetzes von 1849, dessen Revision $z u$ Gunsten einer reaktionären Verfassung gerade diskutiert wurde - noch das Übergewicht hatten, die Konturen einer Arbeiterpolitik aber auch schon sichtbar wurden: die markantesten Programmpunkte sind die Forderung nach Abschaffung der Unterdrückung durch das Kapital (§1), und der Appell an alle Arbeiter, sich miteinander zu solidarisieren (\$1.1)..$^{3}$ Mit dem von Reinholdt Jensen redigierten Folkebladet verfügte der Verein über ein Organ, das zum Forum der frühen Arbeiterpolitik in Dänemark avancierte.

Am 15. September brachte dieses Blatt einen Artikel seines Redakteurs, in dem dieser zur Frage der dänischen Streikbrecher in England Stellung nahm. Er warnte die dänischen Streikbrecher davor, sich dorthin locken zu lassen, denn

„die Schneidergesellen in England haben zur Zeit in grossem Ausmasse die Arbeit niedergelegt, weil die Unternehmer den Arbeitslohn heruntersetzen wollten. Die ausgezeichnete Organisation der Arbeiter in Frankreich, Belgien, Deutschland und der Schweiz macht es jedoch wenig wahrscheinlich, dass es den Agenten der Unternehmer gelingen wird, Arbeiter in diesen Ländern zu gewinnen [...]. Überall in den genannten Ländern sind die Arbeitervereine zu verhindern bestrebt, dass Arbeiter aus dem Schneiderfach zur Arbeitssuche unter den herrschenden Verhält-

1 Ebd., S. 45.

2 Ebd., S. 30f.

3 Programm teilw. gedruckt in En Bygning vi rejser, S. 30. 
nissen nach England gehen, und es ist deshalb wahrscheinlich, dass man wie schon früher Leute in Kopenhagen, Stockholm und Kristiania [d.h. Oslo] [. . .] nach England zu locken versucht, wo sie ohne es zu ahnen zum Schaden sowohl der Interessen ihrer Standesgenossen als auch ihrer eigenen missbraucht werden. Soll es gelingen, den Arbeitern eine bessere Stellung in der Gesellschaft zu verschaffen, dann ist es wichtig, dass die Arbeiter aller Länder für ihr gemeinsames Ziel zusammenarbeiten". ${ }^{1}$

Wir erinnern uns: im Mai des Jahres 1866 hatte der dänische Schneider N. P. Hansen als IAA-Agent unter dänischen Streikbrechern in Edinburgh agitiert. Danach war er in Kopenhagen gewesen; wir wissen, dass er am 18. September wieder in London war, wo er vor dem Generalrat von einem trustworthy agent berichtete, dem er in Kopenhagen getroffen hatte. Am 15. September erschien nun Jensens Artikel, in dem sein Verfasser nicht nur über den Streik der Schneidergesellen berichtete, sondern sich auch über die Massnahmen der IAA zur Abwehr von Streikbrechern gut orientiert zeigte. Zwar könnte Jensen über den Streik auch in der dänischen Presse gelesen haben. Das, was er über die Massnahmen der IAA zur Verhinderung des Exports von Streikbrechern nach England schrieb, seine entscheidene Parteinahme für diese Massnahme, sowie die im Artikel zum Ausdruck kommende von Sympathie getragene Haltung gegenüber der IAA (,,ausgezeichnete Organisation") lassen jedoch auf die Begegnung mit einem wohlorientierten Informanten schliessen. Die Annahme, dass N. P. Hansen dieser Informant war, drängt sich geradezu auf: er kannte die Probleme des Streiks der schottischen Schneidergesellen durch seine Tätigkeit als IAA-Agent, und er war danach in Kopenhagen gewesen, wo er seinen Vertrauensmann getroffen hatte. Mit anderen Worten: der Mann, den Hansen als trustworthy agent for the Association bezeichnete, war Reinholdt Jensen, die Seele des Kopenhagener Arbeitervereines. Der Londoner Generalrat scheint sich von den zu Kopenhagen installierten Kontakten noch mehr erhofft zu haben; am 25. September 1866, also eine Woche nach Hansens Bericht über seinen Aufenthalt in Kopenhagen, wurde der IAA-Mann zum korrespondierenden Sekretär für Dänemark ernannt. Daraus lässt sich schliessen, dass London wenigstens mit der Möglichkeit einer Korrespondenz gerechnet hat. ${ }^{2}$

Ein Jahr später unterzeichnete Hansen als korrespondierender Sekretär für Dänemark (und für Holland) die Einladung zum Lausan-

1 Dok. I. Den Hinweis auf diese Stelle verdanke ich M. Wolfe, der mir freundlicherweise das Manuskript seiner unveröffentlichten Arbeit „De københavnske Arbejderforeningers holdning til Socialismen" (Kopenhagen 1971, masch.) zur Einsicht überliess.

2 The General Council, a.a.O., Bd II, S. 37. 
ner Kongress der IAA vom 2 . bis 8 . September $1867,{ }^{1}$ und angelegentlich dieses Kongresses finden sich auch die ersten Spuren einer Korrespondenz zwischen der IAA und Jensen, bzw. dem Kopenhagener Arbeiterverein: fast auf den Tag genau ein Jahr nach N. P. Hansens Besuch in Kopenhagen gelangte ein leider nicht erhaltener Brief aus Kopenhagen zum Lausanner Kongress. Im Bericht über diesen Kongress heisst es im Oktoberheft des Vorboten von 1867:

„Zu den schon in voriger Nummer erwähnten Beistimmungsadressen und Beglückwünschungen [...] sind dem Präsidenten der Sektionsgruppe deutscher Sprache zur Mittheilung an die Kongressversammlung noch folgende zugegangen: [...] von einem dänischen Arbeiterverein in Kopenhagen, welcher der Zahlung für ,Vorboten'-Abonnements 1 Thaler für Beitrag an die Kongresskosten zufügte". ${ }^{2}$

Und ergänzend dazu heisst es im Januarheft des Vorboten von 1868, in Fortsetzung des Berichtes: „Vize-Präsident Becker las den schon erwähnten Brief der Arbeiter Kopenhagens vor."3 Ohne Zweifel handelt es sich hier um den Kopenhagener Arbeiterverein, denn als Schreiber des Briefes lässt sich mit Sicherheit Reinholdt Jensen ermitteln: im Oktoberheft des Vorboten von 1867 werden in der Rubrik „Empfangsbescheinigung” nämlich „Thlr. 3, wovon Thlr. 1 für Kongresskosten, B. Jensen, Kopenhagen"4 verbucht (,B" ist ein redaktioneller Irrtum für „R" wie Reinholdt).

Aus den Zitaten wird deutlich, dass der Kopenhagener Arbeiterverein unter Reinholdt Jensens Federführung Kontakte zur IAA hatte, in jedem Falle mit dem Zentralkomitee der Sektionsgruppe deutscher Sprache der IAA in Genf und - und dieses Faktum ist für die Vorgeschichte der dänischen IAA-Sektion sehr wichtig - dass der Vorbote nach Kopenhagen gelangte.

An dieser Stelle werden auch die letzten Zweifel daran beseitigt, dass Jensen der Mann war, den Hansen als trustworthy agent bezeichnet hatte. Der Beitrag von einem Thaler zu den Kongresskosten kann als eine Geste gewertet werden, die zeigt, dass die Männer des Kopenhagener Arbeitervereines der IAA zumindest Sympathie entgegenbrachten. Die Datierung der Quittung im Vorboten gibt zu einer weiteren Vermutung Anlass: gehen wir davon aus, dass für zwei Jahresabonnements quittiert wird, dann ergibt sich die Möglichkeit,

1 Der Vorbote, 2. Jahrg. (1867), S. 117.

2 Ebd., S. 153.

a A.a.O., 3. Jahrg. (1868), S. 7.

4 A.a.O., 2. Jahrg., S. 160. 
dass Jensen auf das Blatt sofort nach N. P. Hansens Besuch in Kopenhagen abonnierte. Er müsste es dann ab Oktober 1866 erhalten haben.

Da einerseits der Vorbote weder im Oktober 1866 noch in den darauffolgenden Monaten Jensen ein Abonnement quittierte, es andererseits jedoch unwahrscheinlich erscheint, dass Jensen 1867 für zwei parallellaufende Abonnements bezahlt, lässt sich mit einigem Recht vermuten, dass er im September 1867 ein Abonnement nachträglich, ein anderes im Voraus beglich. Jedenfalls ist der September 1867 das Datum, an dem das Abonnement sowohl zur nachträglichen Bezahlung als auch zur Erneuerung fällig gewesen wäre. (Grundsätzlich war die nachträgliche Bezahlung eines Abonnements auf den Vorboten möglich. ${ }^{1}$ )

Ein Jahr später, in der Septembernummer des Vorboten von 1868, heisst es wieder unter „Empfangsbescheinigungen”: „Thlr. 1, Reinhold, Kopenhagen"..2 Ein Brief Jensens an Becker vom 18. August 1868 bestätigt, dass der genannte „Reinhold” tatsächlich Reinholdt Jensen ist (Dok. II). Der sehr knappe und unpersönliche Brief, der nicht direkt an Becker, sondern an den Vorboten gerichtet ist, annonciert einen Thaler für den - nun schon zu beinahe zwei Drittel abgelaufenen - Jahrgang 1868. Hieraus lässt sich schliessen, dass Jensen für die Zeit vom Oktober 1867 bis einschl. September 1868 bezahlte. Hätte er für die Zeit vom Oktober 1868 bis zum September des nächsten Jahres bezahlen wollen, würde er kaum vom Jahrgang 1868 gesprochen haben. Jensen bezahlte mit anderen Worten auch diesmal den Vorboten nachträglich. Unsere Vermutung, dass er schon sein erstes Abonnement nachträglich bezahlte, wird dadurch unterstützt. Es spricht alles in allem sehr viel dafür, dass er das Blatt seit Hansens Besuch und auf dessen Veranlassung bis zum September 1868 kontinuierlich erhalten hat. Jensens Brief an Becker bezeugt im übrigen, dass sein Verfasser sich noch 1868 für die Arbeiterfrage interessierte. Die dänische Literatur hat ihm dieses Interesse bisher immer mit dem Hinweis abgesprochen, er habe sich ab 1866, aus Enttäuschung über die Revision des Junigrundgesetzes zu Gunsten der reaktionären Verfassung von 1866, gegen die er so entschieden Front bezogen hatte, des Interesses an sozialen und politischen Fragen enthalten. ${ }^{3}$

\section{3. „also muss hier Socialisten sein”}

Die verschiedenen Notizen über Dänemark, die im Laufe der Jahre im Vorboten erschienen waren, erregten auch das Interesse des dänischen

1 Z.B. a.a.O., 3. Jahrg., S. 32, wo ausdrücklich für ,rückständige [. . ] Abonnements" quittiert wird.

2 Ebd., S. 144.

3 So zuletzt W. Markvad, a.a.O., S. 32. 
Sozialisten Niels Lorents Petersen. Dieser aus Kopenhagen stammende Kürschnergeselle war der internationalen und vor allem deutschen Arbeiterbewegung kein Unbekannter: nach seinen eigenen Aussagen war es „1842 in Lausanne in der Schweiz [gewesen], dass [er] zum ersten Mal in einen Arbeiterverein eintrat", 1 ziemlich sicher war er auch "Communist" gewesen;2 am 29. November wurde er Mitglied des Zentralrates des IAA, ${ }^{3}$ zwischen 1860 und 1870 hielt er sich hauptsächlich in Paris auf, wo er an der Organisierung deutscher Arbeiter in einer deutschen Sektion massgeblich beteiligt war. ${ }^{\mathbf{4}}$

Im April 1868 besuchte Petersen seine Vaterstadt Kopenhagen. Der Altsozialist und erfahrene Agitator, dem die Arbeiterbewegung Lebensinhalt geworden war, wollte anscheinend den Aufenthalt in seiner Heimat nicht verstreichen lassen, ohne etwas für die Sache getan zu haben, der er sich verschrieben hatte. Er schickte deshalb einen eiligen Brief an Becker in Genf und erklärte dem alten Freund, er erinnere sich, im Vorwärts einmal von einer Geldsendung gelesen zu haben; dieses Geld sei aus Kopenhagen gekommen, und er schloss daraus: „also muss hier Socialisten sein” (Dok. III), weshalb Becker ihm doch die Adresse dieser Leute mitteilen möge. Der Brief ist undatiert, der Zeitpunkt von Petersens Besuch in Kopenhagen liess sich jedoch an Hand einer Notiz im Sozial-Demokraten ${ }^{5}$ sowie eines noch zu besprechenden Briefes genauer bestimmen. Petersens eigenartiger Lapsus - er meinte den Vorboten, schrieb aber Vorwärts - ist sicherlich eine Reminiszens an den alten Pariser Vorwärts; ähnliche Fehlleistungen sind in

1 Petersen an O. C. Andersen, Paris, 28.12.1872, Andersen-Nachlass, ABA : „det var i 1842, i Lausanne i Sveitz, at jeg for første Gang indtraade i en Arbejderforening - og siden den Tid har jeg ofte [h] aft Leilighed for at deeltage i at understøtte vores Sag". Vgl. auch Petersens Brief an Weitling (s.d.), in: Otto Brugger, Die deutschen Handwerkervereine in der Schweiz 1836-1843, Bern 1932, S. $169 f$.

2 Wenigstens rechnen Wermuth und Stieber ihn dazu, vgl. Die Communisten Verschwörungen, 2. Teil, 1854, S. 95, wo Petersen als einziger Däne (aus dem Königreich) verzeichnet ist.

3 Im Protokoll der Generalratssitzung vom 29.11.1864 heisst es: „The following were then added to the Council: Mr. D. Cornelius [. . . ] and Mr. Petersen on the motion of Mr. Eccarius" (The General Council, Bd I, S. 51). Zu den Mitunterzeichnern der Adresse an Lincoln gehört ein P. Petersen; den gleichen Namen finden wir unter der Adresse an Präsident Johnson (ebd., S. 295). Das Personenverzeichnis im gleichen Band macht diesen P. Petersen zum Peter Petersen (ebd., S. 466). Ein P. oder Peter Petersen unter den bekannteren Persönlichkeiten der IAA ist nicht bekannt. Nach Meinung des Verf. verbirgt sich hinter P. Petersen N. L. Petersen.

"Vergl. dazu Götz Langkau, „Die deutsche Sektion in Paris”, in: IRSH, 17. Jahrg. (1972), S. 103-150.

s Der Social-Demokrat, 4. Jahrg., Nr 51 (29.4.1868), „Briefkasten”. 
Petersens Briefen nicht selten. Hier verstärkt sich der Eindruck von Hast durch diesen Lapsus noch mehr.

Petersen erhielt jedenfalls umgehend Antwort von Becker auf seinen Brief. In dem schon erwähnten Brief, den Petersen am 8. März 1887 aus Genf an O. C. Andersen in Kopenhagen schrieb, erinnerte er den Freund und Gesinnungsgenossen an ihre erste Begegnung: es sei Ende März oder Anfang April 1868 gewesen, dass er nach Kopenhagen gekommen sei. Da er einer Korrespondenz in Beckers Vorboten habe entnehmen können, dass es auch in Dänemark Sozialisten geben müsse, habe er nach Genf geschrieben und um die Adressen dieser Leute gebeten. Becker habe ihm daraufhin Reinholdt Jensens Adresse mitgeteilt, und er, Petersen, habe Jensen dann besucht. Petersen fährt in seinen Erinnerungen weiter fort: sein Kopenhagener Freund V. Holm habe ihm damals mitgeteilt, Reinholdt Jensen sei ihm kein Unbekannter, es sei nicht nötig gewesen, wegen dessen Adresse extra nach Genf zu schreiben. Bei seinem zweiten Besuch bei Jensen, so Petersen, habe er dann O. C. Andersen kennengelernt, den späteren Freund und Adressaten des Briefes. Bei dieser Gelegenheit habe Jensen ihm, Petersen, das Kleeblatt Holm, Andersen und sich selbst als „den ganzen dänischen Sozialismus" (,helle den danske Socialisme”) vorgestellt (Dok. IV).

Aber, so Petersen, ausser den genannten Personen müssten bei der Begegnung mit Jensen noch andere dabeigewesen sein, an die er sich jedoch nicht mehr erinnern könne. Die Männer, an deren Namen sich Petersen noch nach beinahe zwanzig Jahren erinnerte, also der Spielkartenmacher O. C. Andersen, der Goldschmied V. Holm und natürlich Reinholdt Jensen, waren alle Mitglieder im Kopenhagener Arbeiterverein. Von den anderen, an deren Namen sich Petersen nicht mehr erinnern konnte, war der eine vielleicht der aus Deutschland eingewanderte Friseur E. W. Klein. Dieser behauptete nämlich am 21. April 1875 in einem Vortrag vor Kopenhagener Gewerkschaften, er habe schon 1867 mit mehreren Gleichgesinnten einen „kleinen internationalen Klub [gegründet], der in Verbindung zum Ausland gestanden [habe]" (,en lille internationale Klub, der stod i forbindelsen med udlandet"). ${ }^{2}$ Vielleicht liegt hier ein Irrtum vor und Klein meinte in Wirklichkeit das Jahr 1868, evtl. auch 1870, wovon noch die Rede sein wird. Wenn nicht, dann muss diese Behauptung als Prahlerei des sehr exzentrischen und auf sein Renommee bedachten E. W. Klein abgetan werden. ${ }^{3}$

1 Auch W. Markvad führt eine Zeile dieses Briefes an, a.a.O., S. 32.

2 Nach dem Referat im Social-Demokraten, 2. Jahrg., Nr 90 (21.4.1875).

3 Klein hatte während Pios Gefängnisaufenthalts den Vorsitz der Zentralbehörde der Koperhagener Gewerkschaften inne. Nach Pios Rückkehr geriet er 
Was aus Petersens Kontakt zu den Männern aus dem Kopenhagener Arbeiterverein geworden ist, kann nicht mehr festgestellt werden. Nichts deutet darauf, dass sich aus dieser Begegnung allein schon dauerhaftere Kontakte ergeben haben könnten, sie hat jedoch dazu beigetragen, den Männern in Kopenhagen ein genaueres Bild der Arbeiterbewegung zu vermitteln. Die Kontakte zwischen Becker und Jensen scheinen Ende 1868 eingeschlafen zu sein. Der Vorbote gelangte jedenfalls nicht mehr nach Kopenhagen. Sicherlich steht das Einschlafen der Kontakte zu Becker im Zusammenhang mit dem endgültigen Abtreten Reinholdt Jensens von der politischen Bühne. Ende des Jahres 1868 legte er sein Folketingsmandat nieder und nahm in jütländischen Vejle den Posten eines Bahnhofvorstehers an. ${ }^{\mathbf{1}}$ Mit Reinholdt Jensen verschwindet der Kopenhagener Arbeiterverein, von dem sich nach 1868 keine Spuren mehr finden. ${ }^{2}$

\section{An old adherent of the Socialist Party}

Der Mangel an Kontakten zu dänischen Arbeitern wurde anscheinend zuerst in London als unerträglich empfunden. Der Generalrat ergriff deshalb die Initiative und beschloss am 5. April 1870, den altbewährten „Cit. Petersen of the Paris German section” nach Dänemark zu schikken, und zwar mit dem ausdrücklichen Ziel, ,to establish sections or correspondences". ${ }^{3}$ Aus dem Protokoll geht hervor, dass Petersen bei diesem Treffen anwesend war, nicht aber, ob er aus Paris extra herbeizitiert worden war, um nach Kopenhagen gesandt zu werden.

Bei der Generalratssitzung 12. April 1870, also eine Woche später, als Petersen schon auf der Reise nach Kopenhagen war, kamen Jung Bedenken, ob es opportun sei, dass ausgerechnet Petersen nach Kopenhagen geschickt worden sei. Das Protokoll hat seine Bemerkungen festgehalten: „Cit. Jung thought that a little more caution should be used in the future in giving credentials than had been used in the case of Citizen Petersen."4 Die Ursache für Jungs Misstrauen war sicherlich Petersens lassalleanistisches Glaubensbekenntnis (siehe Dok. III). Mit dem ausdrücklichen Hinweis auf Petersens Vertrauenswürdigkeit ("Cit. Petersen was an old adherent of the Socialist Party”) und auf die Notwendigkeit, Kontakte mit Dänemark herzustellen, räumte Eccarius den Einwand denn auch beiseite. ${ }^{5}$

mit diesem in Streit und wurde 1876 aus der dänischen Arbeiterbewegung hinausgedrängt. Dazu Bertolt, Pionerer, a.a.O., S. 165-169, $202 \mathrm{f}$.

1 En Bygning vi rejser, S. 43; auch Dansk Biografisk Leksikon, Bd XI, S. 506f., Artikel Reinhold Jensen.

2 W. Markvad, a.a,O.. S. 32.

3 The General Council, Bd III (1868-1870), S. 223.

4 Ebd., S. 225.

5 Ebd. 
Das Protokoll der Generalratssitzung vom 26. April 1870 verzeichnet den Eingang eines Briefes aus Kopenhagen an Jung in London. ${ }^{1}$ Petersen berichtete in diesem schon von Gustav Jaeckh 1904 veröffentlichten Brief, datierend vom 22. April 1870, in der ihm eigenen Diktion über „die erste Zusammenkunft von 9 Sozialisten [...], die erste Zusammenkunft der Art welche seid dem Bestehen des Königreich Dänemark überhaupt stattgefunden hat". ${ }^{2}$ Dieses Treffen wurde in London etwas euphorisch gleich als "the foundation of a Democratic Society which might become a section of the International"3 begrüsst; davon konnte jedoch nicht die Rede sein. In seinem Brief bat Petersen um Informationsmaterial und kündigte ein weiteres Treffen mit den Männern an, die er als „Sozialisten” bezeichnete. Diese zweite Begegnung war für den folgenden Dienstag anberaumt worden.

Über die bei beiden Begegnungen geführten Gespräche lassen sich keine Einzelheiten ermitteln. Immerhin geht aus Petersens Brief an Jung hervor, dass sich sein Verfasser redlich bemühte, seinen Landsleuten die IAA an Hand ihrer Politik, ihrer Ausdehnung und ihrer Organisation zu schildern. Schon bei diesen Gesprächen, in jedem Falle jedoch kurz nachdem Petersen Kopenhagen wieder verlassen hatte, drängte er seine Landsleute, bald schon einen Versuch zur Gründung einer dänischen IAA-Sektion zu unternehmen. In seinem Brief vom 6 . Dezember 1874 an O. C. Andersen erwähnte er nämlich beiläufig:

,als ich vor 4 oder 5 Jahren [d.h. 1870] an Mundberg schrieb, wir sollten doch einen Versuch machen mit einem Aufruf an die dänischen Arbeiter, was er jedoch ablehnte, hatte ich nicht so ganz Unrecht im Hinblick darauf, dass sich der Sozialismus auch bei uns den Weg bahnen würde, denn es gab zur gleichen Zeit uns unbekannte Männer, die unsere Ansichten teilten und deren Situation in der Gesellschaft ein sicheres Resultat [für einen Organisationsversuch] hätte erwarten lassen" (Dok. V).

Petersen glaubte also, ein Aufruf hätte genügt, um die, wie er meinte, sicherlich vorhandenen unzufriedenen Elemente - unzufrieden, weil von der Gesellschaft benachteiligt - unter den Arbeitern und Handwerksgesellen in Kopenhagen zu mobilisieren und zu organisieren. Aus diesem bei oder nach Petersens Besuch 1870 in Kopenhagen gefassten Plan wurde vorerst nichts, die "9 Sozialisten", allen voran Mundberg, hielten entweder die Zeit für einen organisatorischen Vorstoss noch nicht für gekommen, oder sich selbst für nicht befähigt, ein solches Unternehmen auf sich allein gestellt durchzuführen.

1 Ebd., S. 227.

2 Gustav Jaeckh, a.a.O., S. $229 f$.

3 The General Council, Bd III, S. 227. 
Wer gehörte nun zu den Teilnehmern beim Treffen der neun Sozialisten 1870 in Kopenhagen? Weder in seinem Brief an Jung noch in anderen Briefen wird einer dieser Männer ausdrücklich beim Namen genannt. Immerhin geht schon aus dem Brief von 1870 hervor, dass der Kaufmann Anton Nielsen Mundberg dabei war. Petersen hatte Jung gebeten, an seine Adresse das geforderte Informationsmaterial zu schicken. Auch der 74-er Brief macht deutlich, dass Mundberg eine Hauptrolle gespielt hatte. Sicher waren auch V. Holm und O. C. Andersen wieder mit dabei. Es ist anzunehmen, dass Petersen sich gleich nach seiner Ankunft in Kopenhagen an diese beiden Männer gewandt hat, denn ausser ihnen dürfte er kaum irgendwelche potentiellen Sozialisten in seiner Heimatstadt gekannt haben, und sie werden den Kontakt zu den anderen Teilnehmern beim Treffen hergestellt haben. Unter Umständen war auch E. W. Klein anwesend.

Als dritter kommt noch der Schreiner Theodor Sophus Pihl in Frage. Er ist der einzige, auf den Petersens Angaben im Brief an Jung zutreffen, unter den neun Sozialisten seien auch Männer mit Kenntnissen der deutschen, französischen und englischen Sprache gewesen. Pihl beherrschte diese Sprachen, denn er hatte sie sich während langer Jahre auf der Walze angeeignet: 1861 hatte er Dänemark verlassen, war zuerst in Deutschland gewesen, wo er 1863 in Frankfurt am Main Lassalle reden gehört hatte, dann war er in der Schweiz gewesen, später in Frankreich und in Italien; 1867 war er nach England gegangen, und erst am Anfang des Jahres 1870 war er nach Kopenhagen zurückkehrt. ${ }^{1}$ Nicht zuletzt wegen seiner guten Sprachkenntnisse schickte ihn die dänische IAA-Sektion 1872 auf den Haager Kongress. ${ }^{2}$ Beim 1868-er Treffen mit Petersen kann Pihl also nicht dabeigewesen sein, wohl aber 1870. Für seine Teilnahme bei der Begegnung 1870 spricht ausserdem, dass der Vorbote 1871, und zwar im Aprilheft, also sozusagen ein Jahresabonnement nach Petersens Besuch in Kopenhagen, noch einmal für einen dänischen Abonnenten quittiert, und zwar: „P.F.S., Kopenhagen, 1 Thlr."3 P. könnte für Pihl stehen, F. dürfte ein Erratum für T. wie Theodor sein, S. steht dann für Sophus. Pihl ist unter den aus der Vorgeschichte der dänischen IAA-Sektion bekannten Männern der einzige, dessen Vor- und Zuname mit diesen Initialen in Verbindung gebracht werden können. Doch auch wenn ein

${ }^{1} \mathrm{Zu}$ Sophus Pihl ist die Literatur besonders spärlich; meinen Ausführungen liegt eine Broschüre zu Grunde, die die norwegische Sozialdemokratie zum Gedächtnis an ihren Gründer herausgab: Den demokratiske Arbeiderforening (ed.), Sophus Pihl 1840-1888. Et Mindeblad om den afdøde arbeiderven, Bergen 1888, S. 6 ff.

2 Ebd., S. 10; Mehring, Geschichte der deutschen Sozialdemokratie, Berlin 1960, 2. Teil, S. 418, über die Teilnehmer am Haager Kongress: nur „1 Däne”.

3 Der Vorbote, 6. Jahrg. (1871), S. 64. 
anderer der Abonnent gewesen sein sollte: die Datierung der Quittung spricht wiederum für die Annahme, dass das Abonnement nachträglich bezahlt wurde, da genau ein Jahr zuvor Petersen in Kopenhagen gewesen war. Petersen könnte den Abonnenten bei seinem Besuch auf den Vorboten aufmerksam gemacht haben.

Zu den "9 Sozialisten", denen Petersen 1870 in Kopenhagen begegnet war, könnten also die folgenden Männer gehört haben: O. C. Andersen und V. Holm, Petersens alte Bekannte aus dem Jahre 1868, wahrscheinlich noch E. W. Klein und T. S. Pihl; mit Sicherheit war A. Nielsen Mundberg dabei, und natürlich hat Petersen auch sich selbst zu den neun gezählt. Oluf Bertolt meint, auch der Schuhmacher Christian Hørdum könne dabeigewesen sein. Er stützt sich dabei auf eine Aussage des alten Povl Geleff aus dem Jahre 1921. Geleff war 1877 mit Louis Pio nach USA geflohen, 1921 holte die dänische Sozialdemokratie den alten und kranken Mann in seine Heimat zurück. Bertolt bemerkt allerdings nicht, $\mathrm{zu}$ welcher Gelegenheit und wem gegenüber Geleff diese Behauptung gemacht hat. ${ }^{1}$ Nach Petersens Besuch in Kopenhagen ernannte der Generalrat, wie Petersen schon in seinem Brief an Jung gefordert hatte, wieder einen korrespondierenden Sekretär für Dänemark. Diesmal wurde der Zigarrenmacher Cohn mit dem Amt betraut. ${ }^{2}$

\section{En Arbejder (Louis Pio)}

Der letzte uns bekannte Kontakt zwischen Dänemark und der IAA vor dem Auftreten Louis Pios ist also die Quittung im Aprilheft des Vorboten von 1871. Pios Socialistiske Blade, die ihr Verfasser noch als Anonymus mit „ein Arbeiter” (,en Arbejder”) unterzeichnete, leiteten dann die Epoche der organisierten dänischen Arbeiterbewegung ein: der Sozialismus wurde jetzt einem breiten Publikum von Arbeitern und Handwerkern zugänglich gemacht, das organisatorische Dach wurde avisiert und die politischen Ziele formuliert. Pio, der Mann, von dem die Literatur bislang annahm, dass ihm dieses alles in einer einzigen intellektuellen Kraftanstrengung gelang, konnte dabei an Bestehendes anknüpfen: immerhin gab es seit 1866 einen Kreis von Männern, der sich mit der Arbeiterbewegung beschäftigte. Mit diesem Kreis von potentiellen Sozialisten muss Pio in Kontakt gekommen sein, und zwar im Zeitraum zwischen dem Februar und dem Mai 1871. Im Februar hatte es noch so ausgesehen, als wolle Pio als integrer Postbeamter Karriere machen, denn in diesem Monat heimste er

1 Bertolt, Pionerer, S. 31.

2 Zu Cohn, der u.U. gebürtiger Däne war, vgl. The General Council, Bd IV (1870-71), S. 573. 
höchstes Lob für eine Erfindung zur Verbesserung der dänischen Postkästen ein; im Mai erschien das erste Heft der Socialistiske Blade. $\mathrm{Da}$ wir wissen, dass der Vorbote ganz sicher noch im April nach Kopenhagen gelangt ist, kennen wir eine der Quellen, aus denen Pio sein Wissen geschöpft haben könnte. Für Pios Beeinflussung durch den Vorboten spricht deutlich das positive und mit Detailkenntnissen angereicherte Bild von der "Commune" auf den ersten Seiten von Socialistiske Blade II; der Vorbote, der ab April 1871 laufend über die Pariser Ereignisse berichtete, könnte hier Pate gestanden haben. Eine andere Quelle für Pios Wissen könnte das Informationsmaterial gewesen sein, welches Jung auf Veranlassung Petersens nach Kopenhagen geschickt haben wird.

Daneben trägt Pios Vorgehen deutliche Züge von Petersens Plan aus dem Jahre 1870: die Socialistiske Blade waren der Aufruf, den Petersen schon im Jahre 1870 als eine Art von Fanal für den Beginn der organisierten Arbeiterbewegung in Dänemark vorgeschlagen hatte. Sie zielten genau auf das Potential der schlechtgestellten Arbeiter und Handwerker in Kopenhagen, unter denen Petersen zu Recht Anhänger vermutet hatte. Erst nach dem Aufruf folgte, getreu Petersens Vorschlag, die Organisierung. Wir können annehmen, dass Pio mit der als eine Art von früher Keimzelle anzusehenden Gruppe von neun Männern in Kopenhagen zusammengetroffen ist, dass er ihr Material einsehen konnte und einen alten Plan, der schon in ihren Reihen diskutiert worden war, wiederaufgriff. Daneben scheint die Verbindung des Kopenhagener Sozialistenkreises zu Genf noch weiterbestanden zu haben. Im Juniheft des Vorboten von 1871, also Monate bevor die dänische IAA-Sektion gegründet wurde und noch bevor Pios Socialistiske Blade II erschienen waren, wusste Becker schon von einer IAASektion ,auch in Kopenhagen"3 zu berichten. Mag hier auch der Wunsch der Vater des Gedankens gewesen sein, Becker wusste offensichtlich schon von der bevorstehenden Gründung der dänischen IAA-Sektion, sodass Kontakte zwischen Genf und Kopenhagen bestanden haben müssen.

Zusammenfassend können wir feststellen: Die dänische Arbeiterbewegung, genauer gesagt die dänische IAA-Sektion, hatte eine Vorgeschichte. Diese begann mit dem Zusammentreffen von N. P. Hansen und Reinholdt Jensen 1866 in Kopenhagen. Da Jensen das geistige Zentrum des Kopenhagener Arbeitervereins darstellte, bekamen diese Kontakte auch eine gewisse Breitenwirkung. Von der Korrespondenz zwischen Jensen, bzw. dem Kopenhagener Arbeiterverein, und der IAA

1 Schmidt (ed.), 80 Louis Pio Breve, S. 24f. (10.2.1871).

2 Socialistiske Blade, II, S. 3 f.

${ }^{3}$ Der Vorbote, 6. Jahrg., S. 96. 
wissen wir mit Sicherheit nur von zwei Briefen, von denen der eine, der Brief an den Lausanner Kongress, nicht erhalten geblieben ist. Wir könnten aber konstatieren, dass die IAA von Kopenhagen aus mit Sympathie und Interesse beobachtet wurde. Wichtig ist, dass der $V$ orbote schon ab 1866 in Kopenhagen gelesen wurde. Dieses ist ein unmittelbarer Erfolg von Hansens Besuch in Kopenhagen. 1868 und 1870 wurden die Kontakte zwischen der IAA und den dänischen Arbeitern jeweils erneuert. Petersens Besuch im Jahre 1870 trug offiziellen Charakter, und bei oder kurz nach diesem Besuch wurde zum ersten Mal erwogen, eine dänische IAA-Sektion zu gründen. Louis Pio konnte an diese verschiedenartigen Kontakte zwischen Dänemark und der IAA anknüpfen, Petersens Plan zur Gründung einer IAASektion in Kopenhagen konnte wiederaufgenommen werden. Vor Ende Februar 1871 hatte Pio jedoch keine Verbindung zu den Männern um Andersen und Mundberg, und wie diese Verbindung schliesslich zustandegekommen ist, entzieht sich unserer Kenntnis.

Der rote Faden in den frühen Beziehungen zwischen Kopenhagen und der IAA war der Vorbote. Obwohl die Rolle Beckers im einzelnen nicht zu definieren ist, muss er nähere Kontakte zu den Männern besessen haben, die sich schon vor dem Auftreten Pios auf der politischen Bühne Dänemarks mit dem Thema Sozialismus beschäftigten. Eine Äusserung Beckers im letzten Heft seiner Zeitschrift unterstreicht dieses Faktum, ohne jedoch nähere Auskünfte über das Wann und Wie von Beckers Beziehungen zu den potentiellen dänischen Sozialisten zu geben. Becker schrieb:

„Wenn im Laufe 1865 durch die Propaganda des hiesigen Zentralkomite's deutscher Sprache schon mehrere auswärtige Sektionen gegründet, so half alsdann der ,Vorbote' solche in allen Weltrichtungen, wie folgendes Ortsverzeichniss nachweist, in's Leben zu rufen, in Lausanne, [...] Kopenhagen".2

Becker spielte also eine grössere Rolle in den Beziehungen zwischen Dänemark und der IAA als auf Grund der spärlichen Quellen anzunehmen ist. Dafür spricht auch, dass Pios erste Auslandsreise als "Grossmeister" (,stormester”) der dänischen IAA-Sektion, sozusagen seine Antrittsreise als Sozialistenführer, nicht zu Marx und Engels nach London ging, sondern ihn Ende $1871 \mathrm{zu}$ Becker nach Genf führte. ${ }^{3}$

1 Bertolt behauptet, Pio habe seit dem Frühjahr 1871 mit Becker in Verbindung gestanden. Den Beleg bleibt er schuldig. Pionerer, S. 73.

2 Der Vorbote, 6. Jahrg., S. 178.

3 Vergl. dazu Pios Brief an Becker vom 23.2.1872. Pio, wieder in Kopenhagen, bedankt sich für die freundliche Aufnahme durch Becker; Aufschlüsse über die Verbindung Pio-Becker gibt dieser Brief leider nicht. Der Brief wird aufbewahrt im IISG, Nachlass Becker D II 640. 


\section{DOKUMENTE}

Skrædersvendene i England have fortiden i stor Maalestok nedlagt Arbejdet, da Mestrene have villet nedsætte Arbejdslønnen. Den udmærkede Organisation af Arbejderne i Frankrig, Belgien, Tydskland og Schweiz gjør det lidet sandsynligt, at det vil lykkes Mestrenes Agenter at vinde ny Arbejdere i disse Lande, thi Arbejderne ere der saa oplyste, at de klart nok føle, at en Forringelse i Arbejdslønnen i England meget let vil kunne medføre en Nedsættelse ogsaa paa Fastlandet. Overalt i de nævnte Lande ere Arbejderforeningerne derfor i travl Virksomhed for at forhindre Arbejdere af Skræderprofessionen $i$ at søge til England under de nuværende Forhold, og det er derfor sandsynligt, at man ligesom tidligere vil søge i Kjøbenhavn, Stokholm og Kristiania at lokke Folk, som ere ubekjendte med Forholdene til at gaa til England, hvor de uden at ane det ville blive brugte som Redskab til at skade baade deres Standsfællers og deres egne Interesser. Skal det lykkes at skaffe Arbejderne en forbedret Stilling i Samfundet vil det være af Vigtighed, at alle Landes Arbejdere virke sammen til det fælles Maal hvor dette lader sig gjøre, og vi opfordre derfor danske Skrædersvende, som fortiden ønske at udvandre, til fremfor Alt under de nuværende Forhold ikke at søge til England.

Folkebladet, 15 Sept. 1866, Nr 37, Sp. 5

\section{II}

REINHOLDT JENSEN AN JOH. PH. BECKER

Copenhagen d. 18 $\underline{d}$ August 1868

Hiermit folgt 1 Thl. für den Jahrgang 1868. Das Blatt ist mehrmals ungenügend frankiert gewesen.

Die Expedition „Der Vorbote”

Ergebenst

Reinholdt Jensen

Original

IISG, Nachlass Becker D II 21 


\section{III}

N. LORENTS PETERSEN AN JOH. PH. BECKER

\section{Lieber P. Becker!}

o.O.u.D. [Kopenhagen, April 1868]

In Eile einige Zeilen, ich befinde mir seid einige Zeit in mein Vaterland herum flankiert rechts und links Verwandte besucht $\mathrm{u}$ zwar werde ich jetzt einiges Zeit in Copenhagen verweilen vieleicht nur einige Wochen, jedoch ohne alles Verbindung mit Gesinnungsgenossen - Ich erinere mir dass einmal im Vorwärts die Anmeldung ein Geldsendung wo Copenhagen bemerkt war. also muss hier Socialisten sein. bekantlich gehöre ich zu die Lassaleaner. Jedoch ich sehe ja dass in S. Demokrat ein Aufruf von Internationale Assosation erschienen ist. Ich habe nie ein eigentlichen Unterschied zwischen diese beide Richtungen finden können von prinzipiele Standpunkte aus. Uberhaupt habe ich die Uberzeugung dass Alle Arbeiter eins und dieselbe Intresse haben - Es wäre mir erwünscht weñ du mir eine Addresse mitheilte wo die hiesige Internationalen - oder Ihnen meine Addresse mitheilte aber so bald wie möglich - da gesagt es kan̄ sein dass ich nicht lange hier bleibe so viel für heute. Stets dein Alter

L. Petersen

\section{Adresse L Petersen}

bei Herr Schou Nörregade 16

Copenhagen (2⿺ Bagsal)

Original

IISG, Nachlass Becker D II 623

\section{IV}

N. LORENTS PETERSEN AN O. C. ANDERSEN

Genf d 8 Mars 1887

[. . .] det er ikke 20 Aar siden at vi lærte at kjende Hinanden thid det var Aar 1868 i Begyndelsen af April eller sidst af Mars at jeg kom til Kjøbenhavn - Efter en Correspondent i Bekers Vorbote at slutte maatte der ogsaa være Socialister der men det var mig ikke muligt at opfinde dem, [...]. - Jeg var derfor nødsaget at skrive til Beker i Genf - om Oplysning - og han skikkede mig R Jensens Addresse - Min Ven Holm da han hørte dette sagte da han jalp mig med at opsøge Jensen - Aa Ham kjender vi vel du har ikke havd nødig at skrive til Genf - Ved der andet Besøg hos Jensen (naar jeg ikke tager Feil) 
forestillide han mig en Meningsfælle ved Navne O C Andersen - og og saa vidt Jensen yttrede var det egentlig helle den danske Socialismen - der var vel endnu et paar Andre men og saa videre - jeg kan ikke erindre om jeg egentlig medens mit Ophold dengang kom i Berøring med nogen af de par Andrere - $i$ et hvert tilfælde vo er de Andre med samt Reinold Jensen - som trofaste Meningsfæller? kun En er tilovers fra denne forgangne Tid førend Pios Optræden - Kun En mig bekjendt min gamle Ven O C Andersen - den ældste Socialist i Danmark tiltakker med dette ihvorat det ikke er saa udmærket som dette til mig - dog Fanden fløjter mig - O C Andersen og L N Petersen længe leve! Hura Hura Hura! [...]

Original (Schluss fehlt) (Auszug)

ABA, Nachlass O. C. Andersen

\author{
V \\ N. LORENTS PETERSEN AN O. C. ANDERSEN
}

Charleville d 6 Dec 1874

[...] - og da jeg for 4 eller 5 Aar skrev til Mundberg - vi skulde dog gjøre et Forsøg med et Opraab til de danske Arbejdere - som [h]an afslog - havde jeg ikke saa ganske Udredt - i Hensigt til Haabet at Socialismen ogsaa bante sig Veien i Hjemmet - thid til selvsamme Tid vare flere Mänd i Danmark som delete vores Anskuelser - men som vi ikke kjendte - og vis Stilling i Samfundet berettigte til et sikkert Resultat $-[\ldots]$

Original (Auszug)

ABA, Nachlass O. C. Andersen 